“ C 2019 IEEE. Personal use of this material is permitted. Permission from IEEE must be obtained for all other uses, in any current or future media, including

reprinting/republishing this material for advertising or promotional purposes, creating new collective works, for resale or redistribution to servers or lists, or reuse of any copyrighted component of this work in other works." 


\title{
A Millimeter-Wave GCW-SAR Based on Deramp-on-Receive and Piecewise Constant Doppler Imaging
}

\author{
Yijiang Nan ${ }^{(}$, Xiaojing Huang ${ }^{\circledR}$, Senior Member, IEEE, and Y. Jay Guo ${ }^{\circledR}$, Fellow, IEEE
}

\begin{abstract}
A novel generalized continuous-wave synthetic aperture radar (GCW-SAR) based on deramp-on-receive operating in millimeter-wave frequency is proposed in this article. With deramp-on-receive, the receiver sampling rate is drastically reduced, and the downsampled 1-D raw data can be obtained from the received beat signal. Further adopting piecewise constant Doppler (PCD) imaging in the digital domain, a GCW-SAR image can be easily reconstructed by using the existing frequencymodulated continuous-wave (FMCW) radar system. The effects of deramp-on-receive in PCD imaging are analyzed accordingly. The short wavelength of the millimeter-wave carrier used in the proposed GCW-SAR enables high azimuth resolution as well as a short synthetic aperture, which, in turn, significantly reduces the imaging computational complexity. Simulation and experimental results confirm the advantages of the proposed GCW-SAR.
\end{abstract}

Index Terms-Deramp-on-receive, generalized continuouswave synthetic aperture radar (GCW-SAR), millimeter wave, piecewise constant Doppler (PCD) algorithm.

\section{INTRODUCTION}

$\mathbf{S}$ YNTHETIC aperture radar (SAR) as a high-resolution remote sensing technique has been widely used for earth observation operating in all day and all weather conditions [1]-[3]. Recently, a new type of SAR combined with full-duplex technique and continuous-wave signaling, called generalized continuous-wave SAR (GCW-SAR), has been proposed [4]-[7]. With this GCW-SAR, the slow time in azimuth is no longer adopted and thus some intrinsic limitations in conventional SAR systems, such as range cell migration and minimum antenna area constraints [8], are removed. Both the range and azimuth information of the targets is retrieved jointly from the 1-D received raw data after self-interference cancellation (SIC) at the radio frequency front end [9]-[11]. However, similar to conventional pulsed SAR, the original GCW-SAR still requires a high sampling rate to obtain the 1-D raw data according to the bandwidth of the received signal. For a practical airborne GCW-SAR

Manuscript received June 16, 2019; revised July 29, 2019; accepted August 27, 2019. This work was supported by the Australian Research Council Discovery Project under Grant DP 160101693. (Corresponding author: Yijiang Nan.)

The authors are with the Global Big Data Technologies Center, University of Technology Sydney, Ultimo, NSW 2007, Australia (e-mail: yijiang.nan@student.uts.edu.au; xiaojing.huang@uts.edu.au; jay.guo@uts.edu.au).

Color versions of one or more of the figures in this article are available online at http://ieeexplore.ieee.org.

Digital Object Identifier 10.1109/TGRS.2019.2939004 to achieve a high azimuth resolution, a long synthetic aperture time is required, leading to increased computational cost.

In this article, we propose to implement the GCWSAR based on the deramp-on-receive principle as in a frequency-modulated continuous-wave (FMCW) radar. That is, the received signal is mixed with a replica (reference signal) of the transmitted signal and downconverted to the baseband to produce the beat signal via a low-pass filter (LPF). Hence, the received bandwidth can be drastically reduced [12], [13]. Then, the downsampled 1-D received signal can be obtained from the beat signal. Finally, applying the piecewise constant Doppler (PCD) algorithm, an efficient GCW-SAR implementation is achieved. As we all know, with a conventional FMCW radar, the duration of useful beat signal in a chirp period is reduced as the target distance increases. However, the GCWSAR reconstructs the image by performing cross correlation of the received signals with location-dependent reference signals. The targets are distinguished by the time delays and Doppler frequency shifts of the received signal rather than frequency differences. Hence, with a triangular frequency-modulated waveform, all of the beat signal components over the signaling period can be fully utilized in GCW-SAR to capture the maximum signal power.

As the azimuth resolution is theoretically equal to half of the antenna aperture, which is inversely proportional to the synthetic aperture [8], achieving a high azimuth resolution requires a long synthetic aperture. With the PCD imaging adopted in the GCW-SAR, the slant range is approximated as multiple piecewise linear segments. If the synthetic aperture is long, and/or more accurate approximation is required, the number of linear segments must be increased, leading to increased computational complexity. In order to achieve highresolution imaging with low complexity, we further propose to use millimeter-wave signal in the GCW-SAR. Since the synthetic aperture is proportional to the wavelength of the transmitted signal given the antenna aperture, the proposed millimeter-wave GCW-SAR will have short synthetic aperture once the wavelength is reduced to millimeter scale. With the same high azimuth resolution, a short synthetic aperture not only reduces the computational cost significantly but also improves PCD image quality. Note that the length of the synthetic aperture will not restrict the image size in azimuth since the GCW-SAR imaging in the azimuth direction is a recursive and on-going process. 


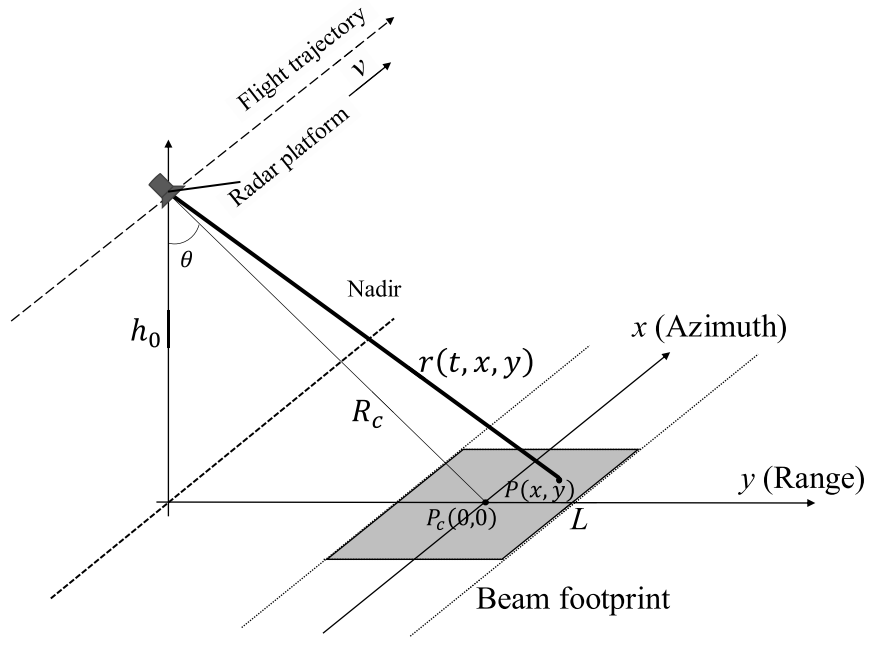

Fig. 1. SAR stripmap geometry.

The remainder of this article is organized as follows. In Section II, the GCW-SAR block diagram with derampon-receive and PCD imaging is described, followed by the discussions on how to select the transmitted signal waveform and how the downsampled received signal impacts on the PCD imaging performance and complexity. In Section III, the millimeter-wave GCW-SAR is proposed and its major advantages are elaborated in detail. Sections IV and V present the simulation and experiment results, respectively, which validate the proposed implementations. Finally, conclusions are drawn in Section VI.

\section{GCW-SAR With DeRAMP-ON-RECEIVE AND PCD}

For simplicity, the GCW-SAR working in the flat-terrain stripmap geometry shown in Fig. 1 is considered in this article, where the side-looking radar, at a height $h_{0}$, travels along the $x$-direction with a constant speed $v$. The main mathematical symbols and their definitions used in this article are given in Table I.

Further assuming that the difference between the forward and backward wave propagations is negligible, the round-trip time delay can be expressed as $((2 r(t, x, y)) / c)$ where the instantaneous slant range is derived as

$$
\begin{aligned}
r(t, x, y) & =\sqrt{\left(R_{c} \sin \theta+y\right)^{2}+(x-v t)^{2}+h_{0}^{2}} \\
& =\sqrt{R^{2}(y)+(x-v t)^{2}} \approx R(y)+\frac{(x-v t)^{2}}{2 R(y)}
\end{aligned}
$$

which is valid under the condition $|x-v t| \ll R(y)=$ $\left(\left(R_{c} \sin \theta+y\right)^{2}+h_{0}^{2}\right)^{1 / 2}$ where $R_{c}$ is the closest range from the flight trajectory to the scene center.

As the GCW-SAR imaging is a cross correlation process between the received signal and the location-dependent reference signal [4], the image of a target located at $\left(x_{m}, y_{n}\right)$ can be expressed as

$$
\begin{array}{r}
I\left(x_{m}, y_{n}\right)=\int_{\frac{x_{m}}{v}-\frac{T}{2}}^{\frac{x_{m}}{v}+\frac{T}{2}} S_{r}(t) s^{*}\left(t-\frac{2 r\left(t, x_{m}, y_{n}\right)}{c}\right) \\
\times e^{j \frac{4 \pi}{\lambda} r\left(t, x_{m}, y_{n}\right)} d t
\end{array}
$$

TABLE I

CW-SAR PARAMETERS

\begin{tabular}{|c|c|}
\hline Symbols & Definitions \\
\hline$t$ & Time variable \\
\hline$r(t, x, y)$ & Slant range from the antenna to an arbitrary point $P(x, y)$ \\
\hline$\sigma(x, y)$ & Backscattering coefficient of the point $P(x, y)$ \\
\hline$R_{c}$ & Closest range from the flight trajectory to the scene center \\
\hline$c, v$ & Speeds of light and radar platform \\
\hline$T_{r}$ & Transmitted chirp signal repetition period \\
\hline$T_{P}$ & Time duration of a linear segment in PCD imaging \\
\hline$T_{s}$ & Sampling interval of received signal \\
\hline$T_{b}$ & Sampling interval of beat signal \\
\hline$P$ & Number of the linear segments in PCD imaging \\
\hline$T$ & Traveling time over the synthetic aperture \\
\hline$L$ & Length of the synthetic aperture \\
\hline$L_{a}$ & Length of the physical antenna aperture \\
\hline$\Delta y, \Delta x$ & Pixel spacings in range and azimuth respectively \\
\hline$n, m$ & Pixel indexes in range and azimuth respectively \\
\hline$\theta$ & Beam incident angle \\
\hline$\lambda, f_{c}$ & Transmit carrier wavelength and frequency respectively \\
\hline$\delta_{x}, \delta_{y}$ & Range and azimuth resolutions respectively \\
\hline
\end{tabular}

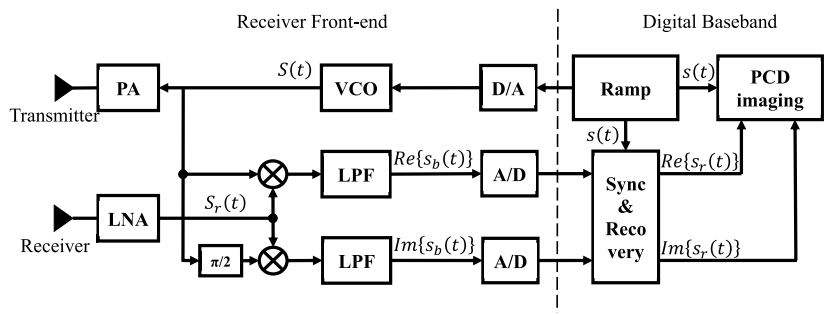

Fig. 2. Block diagram of GCW-SAR with deramp-on-receive, where PA and LNA denote power amplifier and low-noise amplifier, respectively, VCO denotes the voltage-controlled oscillator producing carrier frequency, A/D and D/A are analog-digital and digital-analog converters and Sync represents time synchronization. The downsampled received signal can be obtained after the "Sync \& Recovery" module.

where $s_{r}(t)$ is the received baseband signal, $s(t)$ is the transmitted baseband signal, and $*$ denotes the conjugate operation.

In this section, we first describe the GCW-SAR deramping process, then compare the beat signals produced by different transmitted waveforms, and finally discuss the PCD imaging performance.

\section{A. RF Deramping at Receiver Front End}

The proposed low-complexity GCW-SAR architecture is shown in Fig. 2. Assuming that $s(t)$ has a constant envelope which is also normalized to 1 for simplicity, i.e., $|s(t)|^{2}=$ $s^{*}(t) s(t)=1$, the received baseband signal can be expressed as

$$
s_{r}(t)=s_{r}(t) s^{*}(t) s(t)=s_{b}(t) s(t)
$$

where

$$
s_{b}(t)=s_{r}(t) s^{*}(t)
$$

is the beat signal similar to the one used in an FMCW radar. Expressing the transmitted and received radio frequency signals as $S(t)=\operatorname{Re}\left\{s(t) e^{j 2 \pi f_{c} t}\right\}$ and $S_{r}(t)=\operatorname{Re}\left\{s_{r}(t) e^{j 2 \pi f_{c} t}\right\}$, respectively, $\operatorname{Re}\left\{s(t) e^{j 2 \pi f_{c} t}\right\}$. $S_{r}(t)=(1 / 2) \operatorname{Re}\left\{s_{r}(t) s(t) e^{j 4 \pi f_{c} t}\right\}+(1 / 2) \operatorname{Re}\left\{s_{b}(t)\right\}$ and $\operatorname{Im}\left\{s(t) e^{j 2 \pi f_{c} t}\right\} \cdot S_{r}(t)=(1 / 2) \operatorname{Im}\left\{s_{r}(t) s(t) e^{j 4 \pi f_{c} t}\right\}-$ $(1 / 2) \operatorname{Im}\left\{s_{b}(t)\right\}$. After removing the high-frequency components with carrier frequency $2 f_{c}$ by LPFs, we see that the beat 
signal $s_{b}(t)$ can be obtained by using the $I / Q$ demodulator at the RF front end. As the bandwidth of the beat signal $s_{b}(t)$ is much smaller than the received signal $s_{r}(t)$, only lower sampling rate analog-digital converters (ADCs) are required. With the known transmitted signal frequency ramp and the sampling time of the A/D converters, the downsampled transmitted baseband signal $s(t)$ can be regenerated. Once the beat signal is obtained, the downsampled version of the original received signal can be easily recovered from (3) in digital baseband.

Although passing through the LPFs introduces a time delay to the beat signal, it can be compensated after time synchronization in digital baseband. From the block diagram of the GCW-SAR with deramp-on-receive, we see that the differences between the FMCW radar system and the proposed GCW-SAR are the additional time synchronization and the imaging process in digital baseband. Their RF deramping processes in receiver front end are the same. Therefore, the GCW-SAR with the deramp-on-receive can be easily implemented by using the existing FMCW radar hardware, which can be validated by the deramped GCW-SAR experimental results presented in Section VI.

\section{B. Transmitted Waveform Selection}

The conventional FMCW radar adopts the upchirp/downchirp signal, producing the unwanted highfrequency components in the beat signal. After lowpass filtering, these components are removed so that the corresponding received signal components are lost. In order to completely pass through the LPF, the highest instantaneous frequency of the beat signal must be smaller than the cutoff frequency of the LPF. Therefore, a triangular frequencymodulated waveform is selected for the proposed GCW-SAR, which not only retains the characteristics of the chirp signal but also produces beat signal without high-frequency components. Considering the phase continuity, over the period $\left[0, T_{r}\right)$, the triangular frequency-modulated waveform can be expressed as

$$
s(t)= \begin{cases}e^{j \pi K_{r} t\left(t-\frac{T_{r}}{2}\right)}, & t \in\left[0, \frac{T_{r}}{2}\right) \\ e^{-j \pi K_{r}\left(t-\frac{T_{r}}{2}\right)\left(t-T_{r}\right)}, & t \in\left[\frac{T_{r}}{2}, T_{r}\right)\end{cases}
$$

where $K_{r}=2 B / T_{r}$ is the chirp rate and $B$ is the bandwidth of the transmitted signal.

Fig. 3 depicts the frequency variations of the beat signals with respect to time $t$ produced by the upchirp waveform and the triangular frequency-modulated waveform, respectively. With the upchirp waveform, a high-frequency signal is obtained at the start of each transmitted repetition period due to a sharp frequency transition as shown in Fig. 3(a), and it will be filtered out after passing through a narrow-bandwidth LPF. With the triangular frequency-modulated waveform, there is no sharp frequency transition so that the deramping will never produce high beat frequency as shown in Fig. 3(b).

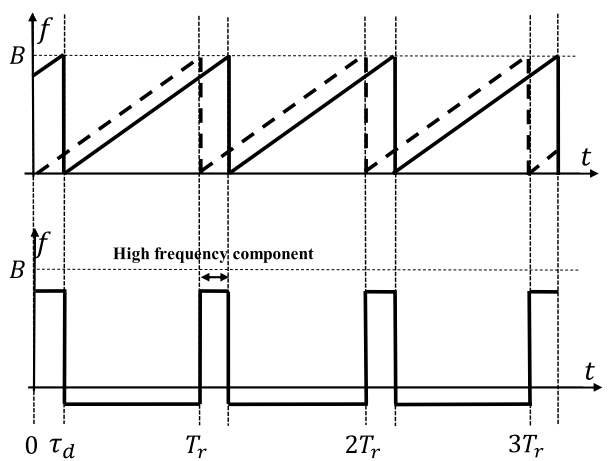

(a)

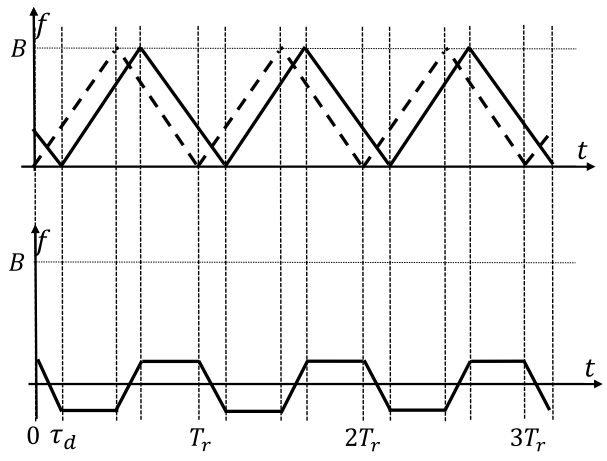

(b)

Fig. 3. Beat frequency produced by (a) upchirp waveform and (b) triangular frequency-modulated waveform, respectively.

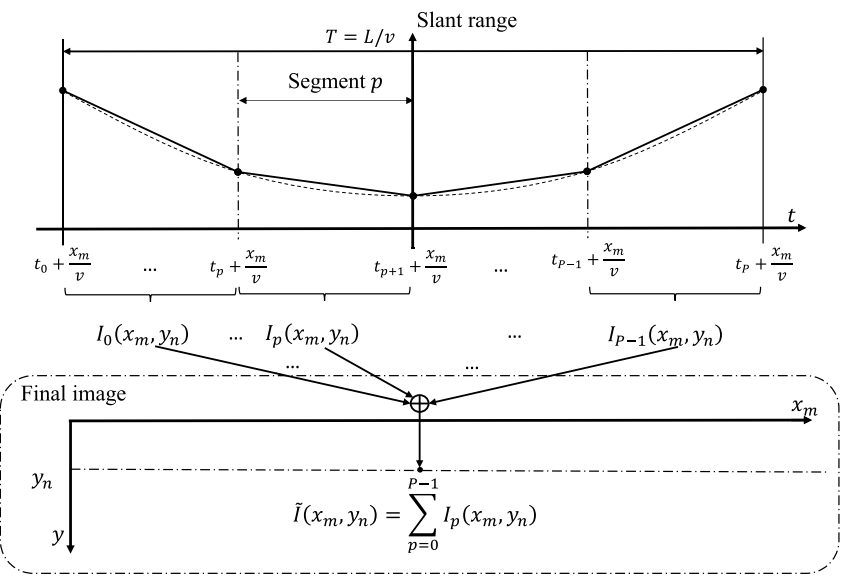

Fig. 4. PCD imaging process.

\section{PCD Imaging in Digital Baseband}

With the PCD algorithm described in [4], the range curve has been approximated as $P$ linear segments linked end to end. Fig. 4 describes the PCD imaging process clearly. Assuming that the interval of the $p$ th segment of the slant range $r\left(t, x_{m}, y_{n}\right)$ is set to $\left[t_{p}+\left(x_{m} / v\right), t_{p+1}+\left(x_{m} / v\right)\right)$ where $t_{p}$ is the time instant at the segment joint points, $t_{p}=p(T / P)-T / 2$ for $p=0,1, \ldots, P-1$, the approximated range curve can be expressed as

$$
\begin{aligned}
\widetilde{r}\left(t, x_{m}, y_{n}\right)= & \frac{r\left(t_{p+1}+\frac{x_{m}}{v}, x_{m}, y_{n}\right)-r\left(t_{p}+\frac{x_{m}}{v}, x_{m}, y_{n}\right)}{\left(t_{p+1}-t_{p}\right)} \\
& \cdot\left(t-t_{p}-\frac{x_{m}}{v}\right)+r\left(t_{p}+\frac{x_{m}}{v}, x_{m}, y_{n}\right)
\end{aligned}
$$




$$
\begin{gathered}
=\frac{r\left(t_{p+1}, 0, y_{n}\right)-r\left(t_{p}, 0, y_{n}\right)}{\left(t_{p+1}-t_{p}\right)}\left(t-t_{p}-\frac{x_{m}}{v}\right) \\
+r\left(t_{p}, 0, y_{n}\right) \\
=-\frac{\lambda}{2} f_{D_{p}}\left(y_{n}\right)\left(t-t_{p}-\frac{x_{m}}{v}\right)+r\left(t_{p}, 0, y_{n}\right) \\
t \in\left[t_{p}+\frac{x_{m}}{v}, t_{p+1}+\frac{x_{m}}{v}\right)
\end{gathered}
$$

where $f_{D_{p}}\left(y_{n}\right)=-(2 / \lambda)\left(\left(r\left(t_{p+1}, 0, y_{n}\right)-r\left(t_{p}, 0, y_{n}\right)\right) /\right.$ $\left.\left(t_{p+1}-t_{p}\right)\right)$ is defined as the constant Doppler frequency shift for the $p$ th segment. Therefore, the final PCD at point $\left(x_{m}, y_{n}\right)$ image can be further derived as

$$
\begin{aligned}
& \widetilde{I}\left(x_{m}, y_{n}\right) \\
& \quad=\int_{\frac{x_{m}}{v}-\frac{T}{2}}^{\frac{x_{m}}{v}+\frac{T}{2}} s_{r}(t) s^{*}\left(t-\frac{2 \widetilde{r}\left(t, x_{m}, y_{n}\right)}{c}\right) e^{j \frac{4 \pi}{\lambda} \widetilde{r}\left(t, x_{m}, y_{n}\right)} d t \\
& \quad=\sum_{p=0}^{P-1} I_{p}\left(x_{m}, y_{n}\right)
\end{aligned}
$$

where

$$
\begin{aligned}
I_{p}\left(x_{m}, y_{n}\right)= & \int_{\frac{x_{m}}{v}+t_{p}}^{\frac{x_{m}}{v}+t_{p+1}} s_{r}(t) s^{*}\left(t-\frac{2 \widetilde{r}\left(t, x_{m}, y_{n}\right)}{c}\right) \\
& \cdot e^{-j 2 \pi f_{D_{p}}\left(y_{n}\right)\left(t-t_{p}-\frac{x_{m}}{v}\right)+j \frac{4 \pi}{\lambda} r\left(t_{p}, 0, y_{n}\right)} d t
\end{aligned}
$$

is the integral over the $p$ th segment. If the transmitted signal satisfies the condition $s^{*}\left(t-\left(\left(2 r\left(t, x_{m}+\Delta x, y_{n}\right)\right) / c\right)\right) \approx$ $s^{*}\left(t-\left(\left(2 r\left(t, x_{m}, y_{n}\right)\right) / c\right)\right)$, the image $I_{p}\left(x_{m}+\Delta x, y_{n}\right)$ at the next point $\left(x_{m}+\Delta x, y_{n}\right)$ with the azimuth spacing $\Delta x$ can be derived as

$$
\begin{aligned}
& I_{p}\left(x_{m}+\Delta x, y_{n}\right) \\
& =\int_{\frac{x_{m}}{v}+\Delta x+t_{p}}^{\frac{x_{m}}{v}+\Delta x+t_{p+1}} s_{r}(t) s^{*}\left(t-\frac{2 \widetilde{r}\left(t, x_{m}+\Delta x, y_{n}\right)}{c}\right) \\
& \cdot e^{-j 2 \pi f_{D_{p}}\left(y_{n}\right)\left(t-t_{p}-\frac{x_{m}+\Delta x}{v}\right)+j \frac{4 \pi}{i} r\left(t_{p}, 0, y_{n}\right)} d t \\
& \approx \int_{\frac{x_{m}}{v}+t_{p}}^{\frac{x_{m}}{v}+t_{p+1}} s_{r}(t) s^{*}\left(t-\frac{2 \widetilde{r}\left(t, x_{m}, y_{n}\right)}{c}\right) \\
& \cdot e^{j \frac{4 \pi}{\lambda}\left(-\frac{\lambda}{2} f_{D_{p}}\left(y_{n}\right)\left(t-t_{p}-\frac{x_{m}}{v}\right)+r\left(t_{p}, 0, y_{n}\right)\right)} e^{j 2 \pi f_{D_{p}}\left(y_{n}\right) \frac{\Delta x}{v}} d t \\
& -\int_{\frac{x_{m}}{v}+t_{p}}^{\frac{x_{m}}{v}+\frac{\Delta x}{v}+t_{p}} s_{r}(t) s^{*}\left(t-\frac{2 \widetilde{r}\left(t, x_{m}, y_{n}\right)}{c}\right) \\
& \cdot e^{j \frac{4 \pi}{\lambda}\left(-\frac{\lambda}{2} f_{D_{p}}\left(y_{n}\right)\left(t-t_{p}-\frac{x_{m}+\Delta x}{v}\right)+r\left(t_{p}, 0, y_{n}\right)\right)} d t \\
& +\int_{\frac{x_{m}}{v}+t_{p+1}}^{\frac{x_{m}}{v}+\frac{\Delta x}{v}+t_{p+1}} s_{r}(t) s^{*}\left(t-\frac{2 \widetilde{r}\left(t, x_{m}, y_{n}\right)}{c}\right) \\
& \cdot e^{j \frac{4 \pi}{\lambda}\left(-\frac{\lambda}{2} f_{D_{p}}\left(y_{n}\right)\left(t-t_{p}-\frac{x_{m}+\Delta x}{v}\right)+r\left(t_{p}, 0, y_{n}\right)\right)} d t \\
& =I_{p}\left(x_{m}, y_{n}\right) e^{j 2 \pi f_{D_{p}}\left(y_{n}\right) \frac{\Delta x}{v}} \\
& -\int_{t_{p}}^{\frac{\Delta x}{v}+t_{p}} s_{r}\left(t+\frac{x_{m}}{v}\right) s^{*}\left(t+\frac{x_{m}}{v}-\frac{2 r\left(t, 0, y_{n}\right)}{c}\right) \\
& \cdot e^{j \frac{4 \pi}{\lambda} \widetilde{r}\left(t, \Delta x, y_{n}\right)} d t \\
& +\int_{t_{p+1}}^{\frac{\Delta x}{v}+t_{p+1}} s_{r}\left(t+\frac{x_{m}}{v}\right) s^{*}\left(t+\frac{x_{m}}{v}-\frac{2 r\left(t, 0, y_{n}\right)}{c}\right) \\
& \cdot e^{j \frac{4 \pi}{\lambda} \widetilde{r}\left(t, \Delta x, y_{n}\right)} d t
\end{aligned}
$$

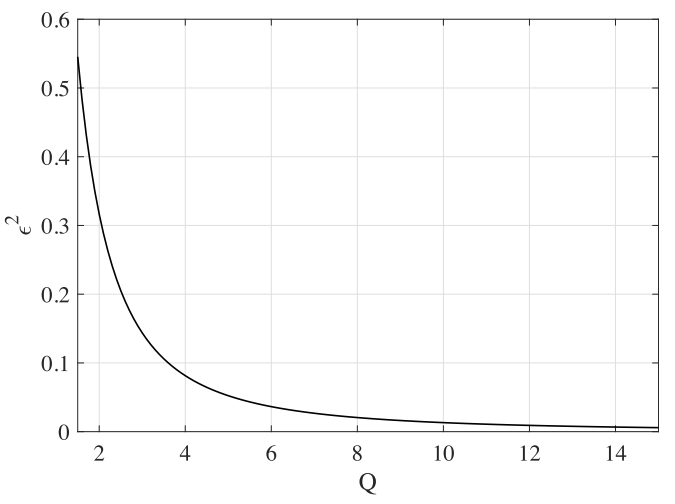

Fig. 5. Relationship between PCD error $\varepsilon^{2}$ and image quality factor $Q$.

which leads to a fast recursive PCD imaging algorithm. In digital baseband, the recursive PCD imaging algorithm can be implemented as

$$
\begin{aligned}
I_{p}(( & \left.m+1) \Delta x, y_{n}\right) \\
= & I_{p}\left((m+1) v T_{s}, y_{n}\right) \\
= & I_{p}\left(m v T_{s}, y_{n}\right) e^{j 2 \pi f_{D_{p}}\left(y_{n}\right) T_{s}}-T_{s} s_{r}\left((m+1) T_{s}+t_{p}\right) \\
& \cdot s^{*}\left((m+1) T_{s}+t_{p}-\frac{2 r\left(t_{p}, 0, y_{n}\right)}{c}\right) e^{j \frac{4 \pi}{\lambda} r\left(t_{p}, 0, y_{n}\right)} \\
& +T_{s} s_{r}\left((m+1) T_{s}+t_{p+1}\right) \\
& \cdot s^{*}\left((m+1) T_{s}+t_{p+1}-\frac{2 r\left(t_{p+1}, 0, y_{n}\right)}{c}\right) \\
& \cdot e^{j \frac{4 \pi}{\lambda} r\left(t_{p+1}, 0, y_{n}\right)} .
\end{aligned}
$$

Without deramp-on-receive, to satisfy the Nyquist-Shannon sampling theorem, the sampling rate $1 / T_{S}$ should be larger than the transmitted bandwidth $B$.

After applying the deramp-on-receive technique to the GCW-SAR, the sampling rate can be significantly reduced due to the much more narrow bandwidth of the received beat signal. Assuming that the sampling interval of the beat signal is defined as $T_{b},(10)$ can be derived as

$$
\begin{aligned}
& I_{p}\left((m+1) \Delta x, y_{n}\right) \\
&=I_{p}\left((m+1) v T_{b}, y_{n}\right) \\
&=I_{p}\left(m v T_{b}, y_{n}\right) e^{j 2 \pi f_{D_{p}}\left(y_{n}\right) T_{b}}-T_{b} s_{r}\left((m+1) T_{b}+t_{p}\right) \\
& \quad \cdot s^{*}\left((m+1) T_{b}+t_{p}-\frac{2 r\left(t_{p}, 0, y_{n}\right)}{c}\right) e^{j \frac{4 \pi}{\lambda} r\left(t_{p}, 0, y_{n}\right)} \\
&+T_{s} s_{r}\left((m+1) T_{b}+t_{p+1}\right) \\
& \cdot s^{*}\left((m+1) T_{b}+t_{p+1}-\frac{2 r\left(t_{p+1}, 0, y_{n}\right)}{c}\right) \\
& \cdot e^{j \frac{4 \pi}{\lambda} r\left(t_{p+1}, 0, y_{n}\right)}
\end{aligned}
$$

where the azimuth spacing $\Delta x$ can be extended from $T_{s} v$ to $T_{b} v$. Based on (11), the impacts of the deramping operation on the PCD imaging performance are elaborated from the range and azimuth directions, respectively, as follows.

1) In the range direction, the images at different $y_{n}$ are reconstructed independently by correlating a locationdependent reference signal with the received signal. According to the pulse compression theory, the range 
ambiguity is determined by the time delay between the reference and received signals. It is easily seen in (11) that the time delay $2 r\left(t_{p}, 0, y_{n}\right) / c$ in PCD is independent of the sampling rate of the received signal, $1 / T_{b}$. Therefore, PCD imaging performance is hardly affected by deramping operation in the range direction.

2) In the azimuth direction, the azimuth imaging spacing in the original GCW-SAR is much shorter than the azimuth resolution since the sampling rate of the received signal is much higher than the Doppler bandwidth, leading to a large amount of redundant imaging points and hence significant computational cost for the PCD implementation. The deramp-on-receive can drastically reduce the sampling rate due to the low-bandwidth beat signal, and the downsampled received signal can be recovered, thus largely improving the imaging efficiency. The azimuth spacing in (11) is extended to $T_{b} v$, and the computational cost is further reduced by $T_{b} / T_{S}$ times.

Note that the recovered downsampled received signal is not suitable to be used in the conventional imaging algorithms based on 2-D (fast time and slow time) raw data structure, e.g., the range-Doppler algorithm [8], [14], since the range compression is achieved in fast-time dimension by the convolution between the received signal and a reference signal. The fast time sampling with low sampling rate would cause significant range ambiguity.

\section{Millimeter-WAVE GCW-SAR}

According to the SAR principle, the range and azimuth resolutions are determined by the transmitted bandwidth $B$ and the physical antenna aperture $L_{a}$, respectively, that is,

$$
\delta_{y}=\frac{c}{2 B \sin \theta}
$$

and

$$
\delta_{x}=\frac{\lambda R_{c}}{2 L}=\frac{L_{a}}{2}
$$

where $\lambda$ denotes the transmitted wavelength. From (13), the synthetic aperture can be expressed as

$$
L=\frac{\lambda R_{c}}{L_{a}}
$$

which is determined by the transmitted wavelength, the physical antenna aperture, and the distance from the target. Assuming the same distance $R_{c}$ and the physical antenna aperture $L_{a}$, the synthetic aperture can be shortened by reducing the transmitted wavelength as shown in (14).

In conventional SAR, the synthetic aperture determines the imaging azimuth dimension since the image is reconstructed by focusing the 2-D raw data matrix. To achieve a large image in azimuth, conventional SAR has to achieve several subimages along the flight trajectory block by block and then performs the image stitching to form a large image, e.g., Mosaic SAR [15], [16]. However, shortening the synthetic aperture obviously increases the number of the subimages in azimuth, hence complicating the data stitching. Therefore, it is difficult for the conventional SAR system to achieve a large image by using the millimeter-wave FMCW radar. In GCWSAR, however, the imaging size in azimuth is independent of the synthetic aperture since the image is reconstructed recursively in azimuth. A large GCW-SAR image in azimuth can be directly achieved without data stitching. This nature allows for millimeter transmitted signal to be used in GCWSAR without restriction in the azimuth dimension.

In this section, the advantages of millimeter-wave GCWSAR system with deramp-on-receive are elaborated in various aspects, including high PCD image quality, low computational complexity, and high-range and azimuth resolutions.

\section{A. High Image Quality}

The PCD image quality can be evaluated by the PCD imaging error derived as

$$
\begin{aligned}
\varepsilon^{2} & =\frac{\int_{-\infty}^{\infty}\left|\widetilde{I}\left(x_{m}, 0\right)-I\left(x_{m}, 0\right)\right|^{2} d x_{m}}{\int_{-\infty}^{\infty}\left|I\left(x_{m}, 0\right)\right|^{2} d x_{m}} \\
& =2-2 \operatorname{Re}\left\{\sqrt{\frac{2 Q}{\pi}} e^{j \frac{\pi}{2 Q}}\left(C\left(\sqrt{\frac{\pi}{2 Q}}\right)-j S\left(\sqrt{\frac{\pi}{2 Q}}\right)\right)\right\}
\end{aligned}
$$

where $Q=\left(\left(L_{a} P^{2}\right) / L\right)$ is defined as the image quality factor, and $C($.$) and S($.$) are the Fresnel integrals, respectively. The$ detailed derivation of (15) is provided in the Appendix. The PCD imaging error becomes smaller as $Q$ increases, indicating that a larger $Q$ value leads to a better PCD image quality as shown in Fig. 5. From (21), the PCD image quality factor can be further expressed as

$$
Q=\frac{L_{a}^{2} P^{2}}{\lambda R_{c}}=\frac{L_{a}^{2} P^{2} f_{c}}{c R_{c}} .
$$

We see that the shorter the wavelength of the transmitted signal is, the larger the quality factor is. With the same $L_{a}$ and $P$, adopting the millimeter-wave transmitted signal can achieve a high image quality.

\section{B. Low Complexity}

The GCW-SAR adopts PCD imaging algorithm which approximates the range curve as piecewise linear segments. A long synthetic aperture will increase the number of the linear segments. Given the same image quality and resolutions, the number of linear segments $P$ can be significantly reduced by using a shorter wavelength transmitted signal as shown in (16), thus leading to a low computational complexity and reduced memory requirement. Assuming the same observed scene where the number of range pixels is $N$, the computational complexity of the millimeter-wave GCW-SAR and its required memory are compared with those of the conventional one as follows.

The PCD algorithm updates the image in azimuth recursively whenever the system obtains a new received sample. Assuming that the number of the received samples in the conventional GCW-SAR is $N \times N$, an $N \times N^{2}$ pixel image over the observed scene is achieved. Referring to [4], imaging each pixel needs $3 P+2$ complex multiplications, and thus 


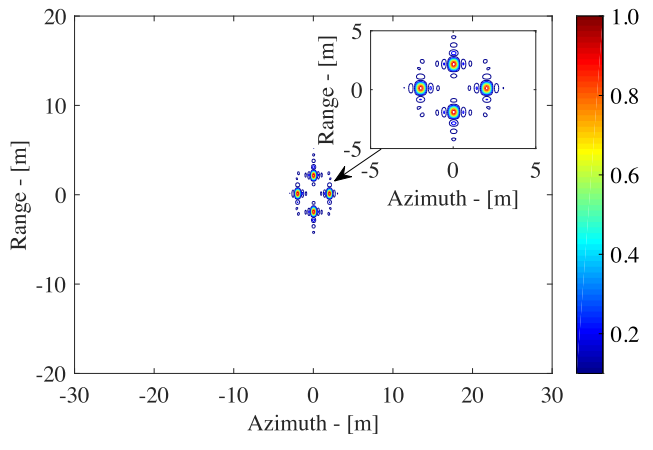

Fig. 6. GCW-SAR with deramp-on-receive imaging result.

$(3 P+2) \times N^{3}$ complex multiplications are required in the conventional GCW-SAR. However, after adopting the millimeterwave transmitted signal with shortened synthetic aperture, the deramp-on-receive can decrease the number of the received samples to $N \times N /\left(T_{b} / T_{s}\right)$. Hence, the computational cost is significantly reduced. Assuming that the number of the linear segments is further reduced to $P / N_{P}$, the proposed GCW-SAR system only requires $\left(3 P / N_{P}+2\right) \cdot N^{2} \cdot N /\left(T_{b} / T_{s}\right)$ complex multiplications.

Assuming that the number of received samples over a transmitted period is $N_{\text {period }}$ and the number of the range pixels is $N$, the number of memory units used in the PCD imaging of the conventional GCW-SAR is equal to $N_{\text {period }}+$ $N \times(3 P+2)$, referring to [4, Fig. 3]. In the proposed GCWSAR, $N_{\text {period }}$ and $P$ have been reduced to $N_{\text {period }} /\left(T_{b} / T_{s}\right)$ and $P / N_{P}$, respectively, and thus the number of memory units is $N_{\text {period }} /\left(T_{b} / T_{s}\right)+N \times\left(3 P / N_{P}+2\right)$.

\section{High Image Resolutions}

As shown in (12) and (13), the range and azimuth resolutions are determined by the transmitted signal bandwidth and the antenna aperture, respectively. Using the millimeter-wave signal allows a wider transmitted signal bandwidth and hence a higher range resolution. On the other hand, a high azimuth resolution requires a short-antenna aperture, which leads to a long synthetic aperture and increases the computational cost. Based on (14), the synthetic aperture can be shortened by using the millimeter-wave radar. Given the high image quality and the low computational cost, a higher azimuth resolution $L_{a} / 2$ can be achieved by transmitting a shorter transmitted wavelength as shown in (16). Therefore, the millimeter-wave FMCW radar can provide high image resolutions in both range and azimuth.

\section{Simulation Results}

In this section, the comprehensive performance evaluation of the novel GCW-SAR and comparison with the millimeterwave FMCW-SAR are made through simulation in an airborne stripmap SAR geometry with the following parameters: platform altitude of $500 \mathrm{~m}$, speed of radar platform of $45 \mathrm{~m} / \mathrm{s}$, the transmitted bandwidth of $250 \mathrm{MHz}$, and $R_{c}=1113.3 \mathrm{~m}$. The noise at the receiver is not considered for simplicity.

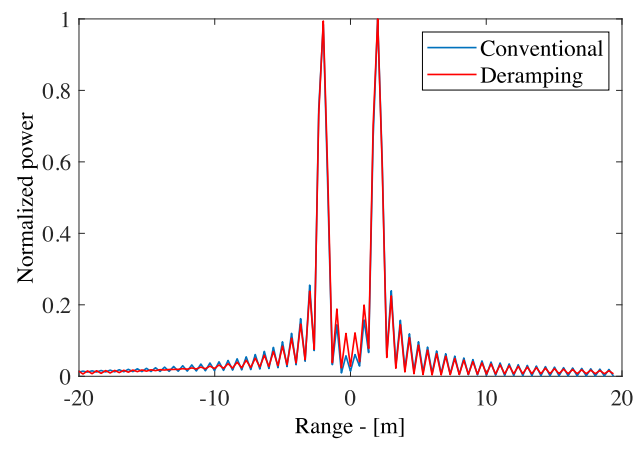

(a)

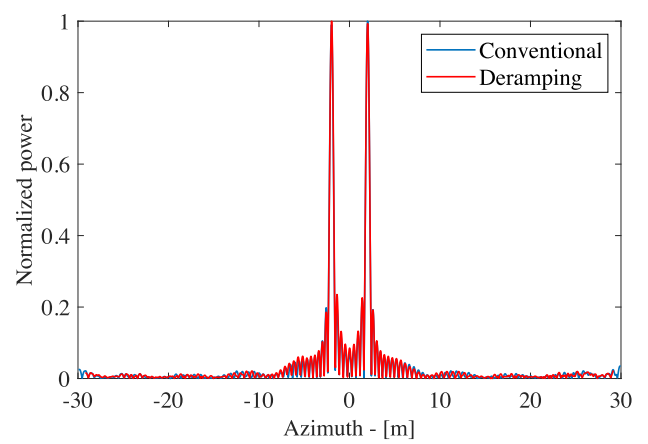

(b)

Fig. 7. Imaging performance comparison between conventional GCW-SAR and GCW-SAR based on deramp-on-receive. (a) Range direction. (b) Azimuth direction.

\section{A. Performance of GCW-SAR Imaging from Beat Signal}

Deramp-on-receive can significantly reduce the sampling rate of the received signal. In the first simulation, the impact of the deramping operation on PCD imaging performance is investigated. The point targets are located at $(-2 \mathrm{~m}, 0)$, $(2 \mathrm{~m}, 0),(0,-2 \mathrm{~m})$, and $(0,2 \mathrm{~m})$. The antenna aperture $L_{a}$ is $0.8 \mathrm{~m}$, the carrier frequency is $7 \mathrm{GHz}$, the number of linear segments $P$ is 40 , and the triangular frequencymodulated waveform is adopted. The full-imaging result is shown in Fig. 6, and the range and azimuth imaging results at $x_{m}=0$ and $y_{n}=0$ are shown in Fig. 7, respectively. It is evident from the imaging results shown in Fig. 7 that the deramp-on-receive in GCW-SAR system does not cause any performance degradation. As the range ambiguity is determined by the time delay of the reference signal with PCD imaging, reducing the samples in the received signal does not affect the range resolution, as shown in Fig. 7(a). In azimuth, reducing the received samples improves the imaging efficiency without azimuth resolution degradation, as shown in Fig. 7(b).

\section{B. High Image Quality by GCW-SAR With Millimeter-Wave Signal}

In the second simulation experiment, we investigate the impact of changing carrier frequency on the image quality of the GCW-SAR. With the triangular frequency-modulated waveform, an image with a point target at $(0,0)$ is considered. The antenna aperture $L_{a}$ is $0.4 \mathrm{~m}$, and the number of linear segment $P$ is 20 . The image quality factor $Q$ as a function of 


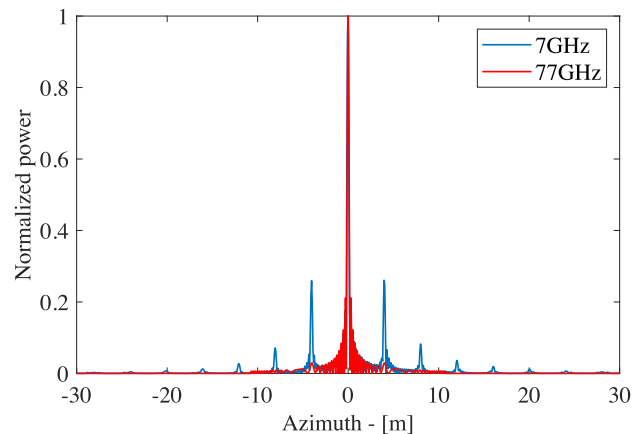

Fig. 8. Azimuth imaging comparison using $f_{c}=7 \mathrm{GHz}$ and $f_{c}=77 \mathrm{GHz}$ when $L_{a}=0.4 \mathrm{~m}$ and $P=20$.

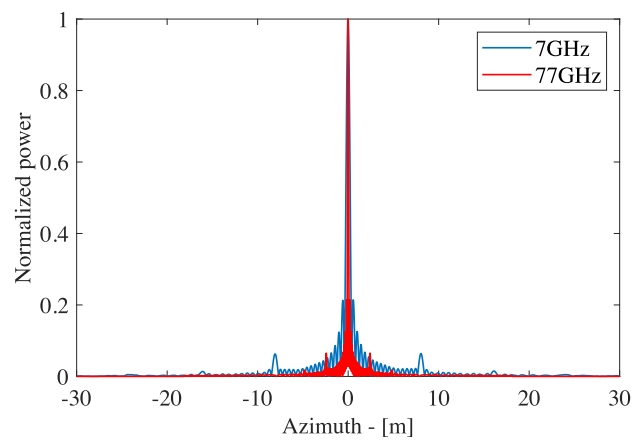

Fig. 9. Azimuth imaging comparison using $f_{c}=7 \mathrm{GHz}$ and $f_{c}=77 \mathrm{GHz}$ when $Q=5.39$ and $P=20$.

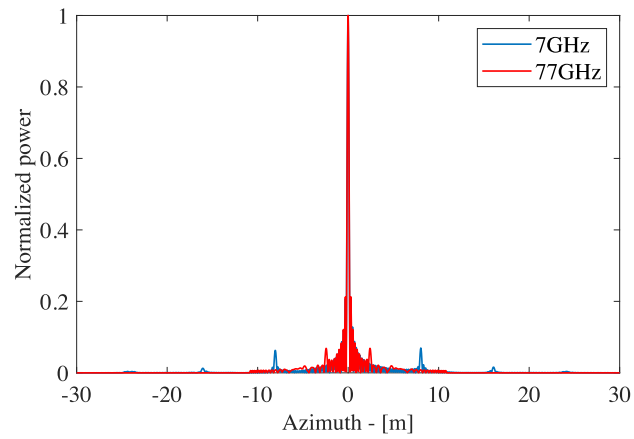

Fig. 10. Azimuth imaging comparison using $f_{c}=7 \mathrm{GHz}$ and $f_{c}=77 \mathrm{GHz}$ when $L_{a}=0.4 \mathrm{~m}$ and $Q=5.39$.

the carrier frequency has been described in (16), where $Q$ is proportional to the carrier frequency. The images in azimuth with 7- and 77-GHz carrier frequencies, respectively, are given in Fig. 8. The results show that the $77-\mathrm{GHz}$ millimeterwave radar can significantly improve the image quality from 1.34 to 14.81 compared with the $7-\mathrm{GHz}$ radar. With $7-\mathrm{GHz}$ carrier frequency, $P=20$ is not enough to approximate the slant range over the 119-m synthetic aperture. However, with $77-\mathrm{GHz}$ carrier frequency, the synthetic aperture is shortened to $11 \mathrm{~m}$, thus drastically reducing the error of slant range approximation. Moreover, assuming that the echoes reflected from any two targets whose spacing is larger than twice synthetic aperture are independent, the interference from the sidelobes can also be suppressed by shortening the synthetic aperture. As shown in Fig. 8, with 77-GHz carrier frequency,

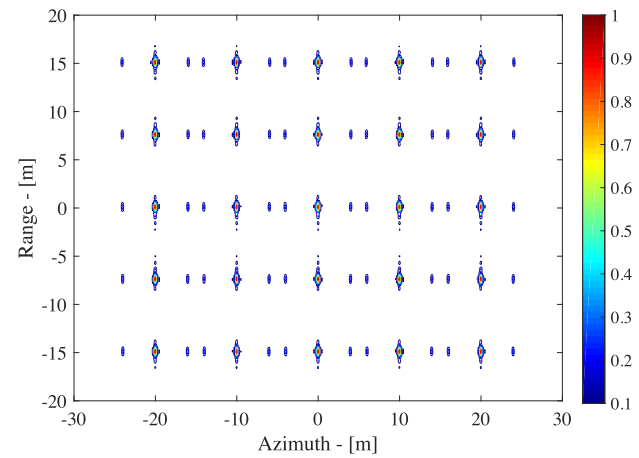

(a)

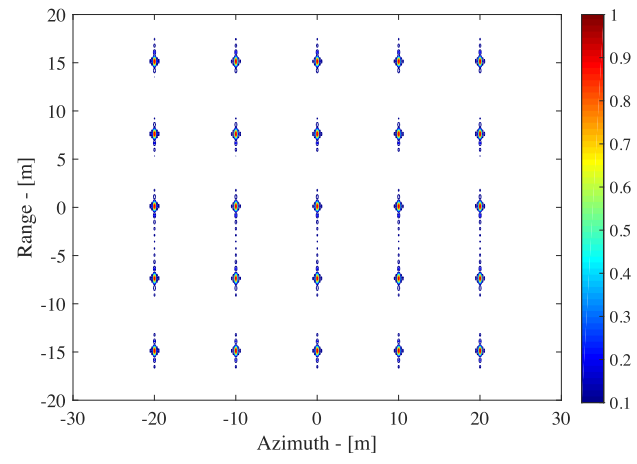

(b)

Fig. 11. Multi-point imaging performance comparison between (a) 7-GHz GCW-SAR and (b) 77-GHz GCW-SAR, where $L_{a}=0.4 \mathrm{~m}$ and $B=$ $250 \mathrm{MHz}$.

the sidelobes can only impact on the image within the interval $(-11 \mathrm{~m}, 11 \mathrm{~m})$.

\section{High Azimuth Resolution by GCW-SAR With Millimeter-Wave Signal}

In the third simulation experiment, given the same $Q$ and $P$, we compare the azimuth resolution by using different carrier frequencies. Assume that $Q$ is 5.39 and $P$ is 20. Adopting the triangular frequency-modulated waveform and an image with one point target at $(0,0)$, the azimuth imaging results with 7- and 77-GHz carrier frequencies, respectively, are provided in Fig. 9, which shows that a higher azimuth resolution image with the same $Q$ and $P$ can be achieved with the increase of the carrier frequency. Maintaining the same $Q=5.39$ and $P=20$, a $0.12-\mathrm{m}$ azimuth resolution can be achieved when transmitting $77-\mathrm{GHz}$ millimeter wave, while transmitting 7-GHz signal can only achieve a $0.4-\mathrm{m}$ azimuth resolution.

\section{Low Complexity by GCW-SAR With Millimeter-Wave Signal}

The impact of changing carrier frequency on the number of linear segments in GCW-SAR is investigated in this simulation experiment. $L_{a}$ is set to $0.4 \mathrm{~m}$. The triangular frequencymodulated waveform is adopted. Given the same $Q=5.39$ and $L_{a}=0.4 \mathrm{~m}$, an image with one point target at $(0,0)$ is obtained by using the 7- and $77-\mathrm{GHz}$ carrier frequencies, respectively, as shown in Fig. 10. To achieve the same image 


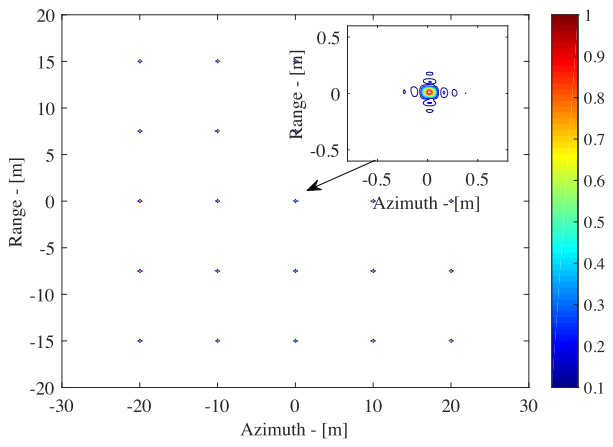

Fig. 12. Multi-point 77-GHz GCW-SAR imaging with high-range and azimuth resolution where $L_{a}=0.2 \mathrm{~m}$ and $B=2.5 \mathrm{GHz}$.

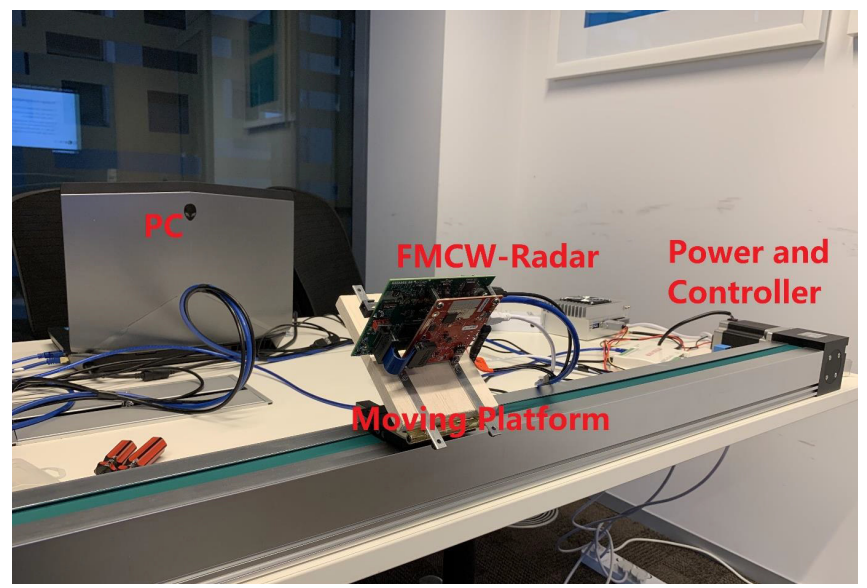

Fig. 13. GCW-SAR laboratory system setup.

quality $Q=5.39, P$ must be as large as 40 in the $7-\mathrm{GHz}$ imaging but only 12 in the $77-\mathrm{GHz}$ imaging, thus saving much computational cost.

\section{E. Multi-Point GCW-SAR Imaging With Millimeter-Wave Radar}

In the final simulation experiment, we compare the multipoint GCW-SAR imaging between the 7-GHz system and the 77-GHz millimeter-wave system. $L_{a}$ and $P$ are set to $0.4 \mathrm{~m}$ and 20 , respectively, and the triangle frequency-modulated waveform is adopted in the two imaging scenarios. By transmitting the $250-\mathrm{MHz}$ bandwidth signals, the reconstructed 7- and 77-GHz images are shown in Fig. 11(a) and (b), respectively. It is evident that $77-\mathrm{GHz}$ millimeter-wave GCWSAR performs much better than the $7-\mathrm{GHz}$ one. To achieve higher range and azimuth resolutions, a wider bandwidth $2.5 \mathrm{GHz}$ and a shorter antenna aperture $L_{a}=0.2 \mathrm{~m}$ are further used in the 77-GHz millimeter-wave GCW-SAR. To keep the same image quality $Q, P$ is increased to 40 and the imaging result is shown in Fig. 12, which validates the capability of the 77-GHz GCW-SAR system to achieve higher imaging resolutions.

\section{EXPERIMENTAL GCW-SAR IMAGING RESUlTS}

To further validate the proposed implementation, we have built the real experimental GCW-SAR imaging system with

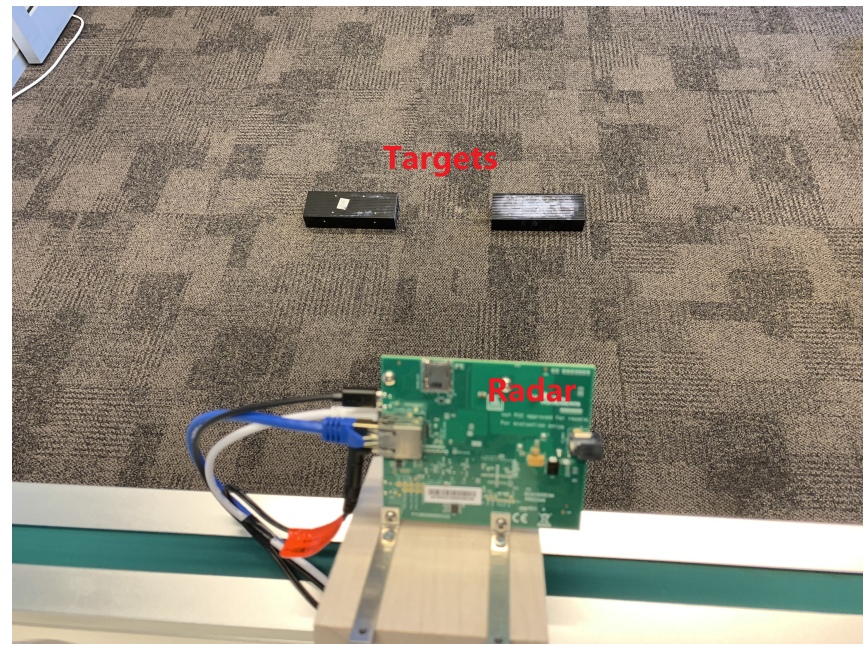

Fig. 14. Radar front view during the measurement.

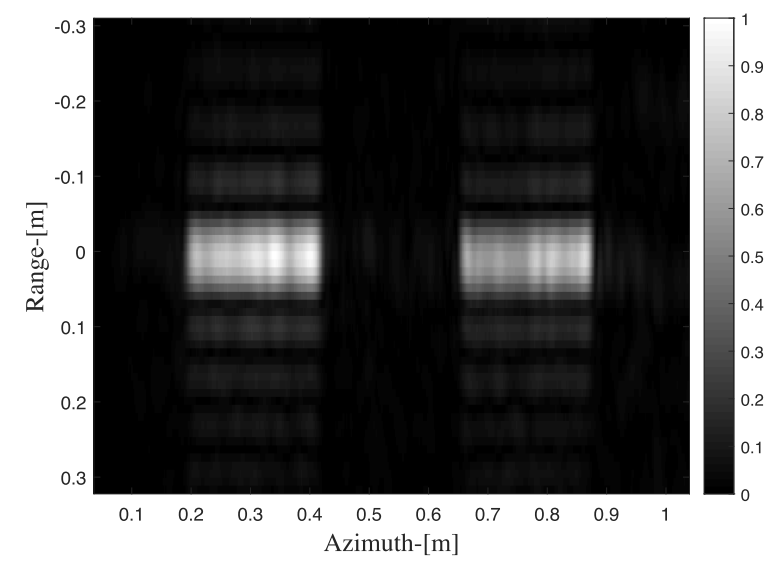

Fig. 15. Real GCW-SAR image.

the millimeter-wave FMCW radar. The system setup and experimental results are presented in this section.

\section{A. Experimental System Setup}

The experimental system is built based on the block diagram of the GCW-SAR with deramp-on-receive as shown in Fig. 2, where the receiver front end uses the AWR1843 single-chip 77-GHz FMCW radar sensor made by Texas Instruments [17], and the imaging processing in the digital domain is performed by a personal computer (PC).

Fig. 13 shows the basic components of the GCW-SAR system. The moving platform controller and the AWR1843 radar sensor are connected to the PC via two micro universal serial bus (USB) ports, respectively. After setting up the radar signal parameters as well as the speed, start, and end locations of the platform, the moving platform and the FMCW radar are triggered when the PC sends command strings to them. The AWR1843 radar is also connected to the PC via an Ethernet cable, by which the received data stream is transferred to the PC. In the experiment, the 1-D raw data are obtained by using single-input-single-output antenna working mode with signal bandwidth $3.6 \mathrm{GHz}$ to maximize the range resolution. 


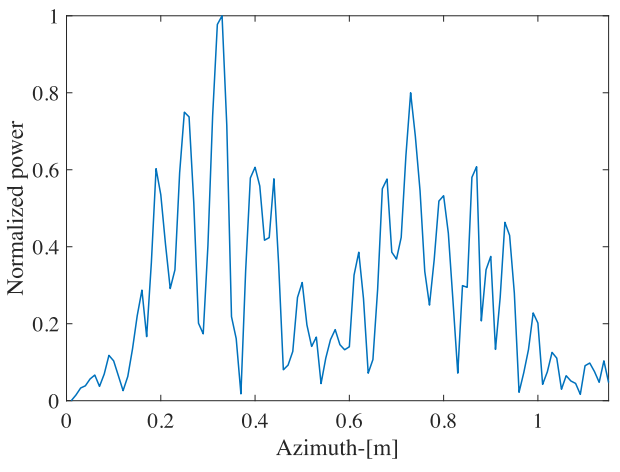

(a)

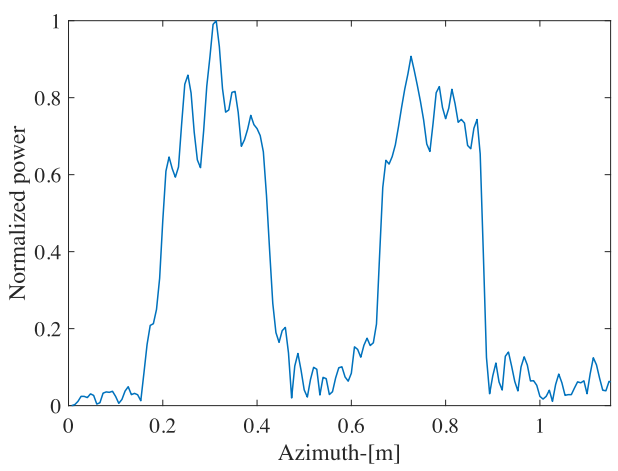

(b)

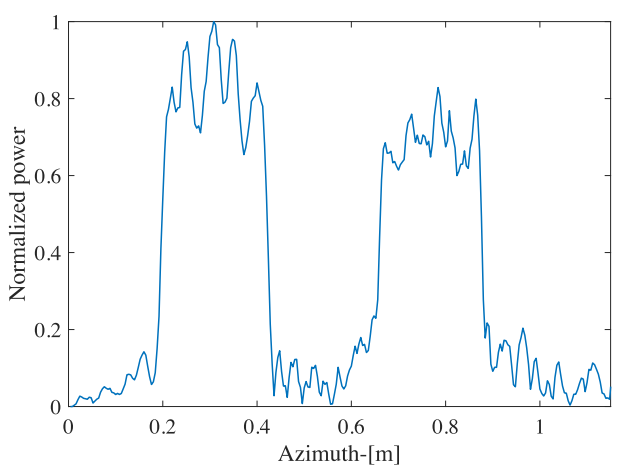

(c)

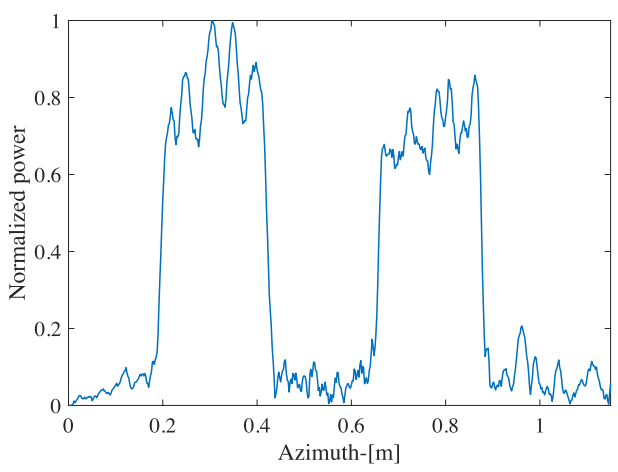

(d)

Fig. 16. Real GCW-SAR image in azimuth with different $Q$ when $y_{n}=0 \mathrm{~m}$. (a) $Q=0.5617$. (b) $Q=1.2638$. (c) $Q=3.5106$. (d) $Q=14.0425$.

Two targets of rectangular metal blocks are measured with dimension $0.225 \mathrm{~m} \times 0.08 \mathrm{~m}$. Fig. 14 shows the radar front view during the measurements, where the length in azimuth of the target is $0.225 \mathrm{~m}$. The height of the radar $h_{0}$ is set to $0.92 \mathrm{~m}$, the platform speed is $0.8 \mathrm{~m} / \mathrm{s}$, and the distance from the radar to beam center $R_{c}$ is $1.44 \mathrm{~m}$. Since the AWR1843 radar sensor is not capable of generating the triangular frequency-modulated waveform and its ADC does not work whenever the beat signal is at high frequencies, the 1-D beat signal is obtained by concatenating the received samples in all chirp periods and padding zeros during ADC idle time.

\section{B. Experimental Results}

After obtaining the beat signal from the AWR1843 radar sensor, the downsampled 1-D received signal is recovered based on (3) and synchronized with the knowledge of the transmitted signal. Without deramp-on-receive, the sampling rate must be larger than $3.6 \mathrm{GHz}$ to satisfy the NyquistShannon sampling theorem. After the deramping, the sampling rate is reduced to $10 \mathrm{MHz}$, and thus the azimuth spacing is extended by 360 times, saving much computational cost. Assuming that $P$ is set to 10 and the synthetic aperture $L$ is set to $0.2 \mathrm{~m}$, the final GCW-SAR image is obtained as shown in Fig. 15, where the range and azimuth resolutions are 0.054 and $0.014 \mathrm{~m}$, respectively. It is evident that the shape of the two targets can be reconstructed accurately compared with Fig. 14, thus validating the PCD algorithm in a practical GCW-SAR system and the deramping operation. The range ambiguities seen in the reconstructed image are the sidelobes caused by the worse range resolution, which is determined by the signal bandwidth. If a larger transmitted bandwidth is available, the range imaging performance can be improved. Since the GCW-SAR imaging is a recursive process, the size of the final image in azimuth is not limited by the synthetic aperture. Note that the SNR of GCW-SAR image can be further improved by using triangular frequencymodulated waveform since the duty cycle of the chirp signal in the experiment can be increased from $70.42 \%$ to $100 \%$.

To further illustrate the PCD algorithm performance in the proposed millimeter-wave GCW-SAR system, the image in azimuth at range $y_{n}=0 \mathrm{~m}$ is reconstructed using different parameters. Based on the PCD imaging error function (15), the image quality factor $Q$ determines the PCD imaging performance, where a larger $Q$ leads to a better image quality. The carrier frequency of the AWR 1843 radar sensor is $77 \mathrm{GHz}$ and $L$ is set to $0.2 \mathrm{~m}$. Hence, $Q$ can be determined by the number of linear segments $P$. Fig. 16 shows the GCWSAR images in azimuth with different $P=2,3,5$, and 10 , where the corresponding $Q=0.5617,1.2638,3.5106$, and 14.0425, respectively. We can see that the GCW-SAR imaging performs better with an increase of $Q$. When $Q$ is larger than 3.5106 , the corresponding PCD error $\varepsilon^{2}<0.1$, which is set as the benchmark to determine the GCW-SAR parameters. The selection of $P, L$, and $\lambda$ should satisfy $Q>3.5106$ based on (16). By using millimeter-wave radar, an image with $Q=3.5106$ and $\delta_{x}=0.014 \mathrm{~m}$ can be achieved when $P=5$, which validates that the proposed GCW-SAR can achieve high quality and high azimuth resolution at low complexity. 


\section{CONCLUSION}

We have shown that with deramp-on-receive front end and PCD imaging algorithm, the GCW-SAR not only works well with downsampled received signal but can also be easily implemented with existing FMCW radar hardware. The shortened synthetic aperture due to the use of millimeterwave signaling results in a number of advantages, such as improved image quality, high imaging resolutions, and reduced computational complexity. The work presented in this article validates a practical GCW-SAR implementation. It is believed that the proposed millimeter-wave GCW-SAR will find wide applications because of its high resolution and low-complexity imaging capability.

\section{APPENDIX}

\section{DERIVATION OF PCD IMAGING ERROR}

For simplicity, assuming that the target is located at the origin $(0,0)$, the received signal can be expressed as $s_{r}(t)=$ $\sigma(0,0) s(t-((2 r(t, 0,0)) / c)) e^{-j(4 \pi / \lambda) r(t, 0,0)}$. From (2) and (7), the GCW-SAR images $I\left(x_{m}, 0\right)$ and $\widetilde{I}\left(x_{m}, 0\right)$ by ideal matched-filtering method and PCD algorithm can, respectively, derived as

$$
\begin{aligned}
& I\left(x_{m}, 0\right) \\
& =\sigma(0,0) \int_{\frac{x_{m}}{v}-\frac{T}{2}}^{\frac{x_{m}}{v}+\frac{T}{2}} s\left(t-\frac{2 r(t, 0,0)}{c}\right) \\
& \quad \cdot s^{*}\left(t-\frac{2 r\left(t, x_{m}, 0\right)}{c}\right) e^{j \frac{4 \pi}{\lambda}\left(r\left(t, x_{m}, 0\right)-r(t, 0,0)\right)} d t \\
& =\sigma(0,0) \int_{-\frac{T}{2}}^{\frac{T}{2}} s\left(t+\frac{x_{m}}{v}-\frac{2 r\left(t+\frac{x_{m}}{v}, 0,0\right)}{c}\right) \\
& \quad \cdot s^{*}\left(t+\frac{x_{m}}{v}-\frac{2 r(t, 0,0)}{c}\right) e^{j \frac{4 \pi}{\lambda}\left(r(t, 0,0)-r\left(t+\frac{x_{m}}{v}, 0,0\right)\right)} d t \\
& \approx
\end{aligned}
$$

and

$$
\begin{aligned}
\widetilde{I}\left(x_{m}, 0\right) & \sigma(0,0) \int_{\frac{x_{m}}{v}-\frac{T}{2}}^{\frac{x_{m}}{v}+\frac{T}{2}} s\left(t-\frac{2 r(t, 0,0)}{c}\right) \\
= & s^{*}\left(t-\frac{2 \widetilde{r}\left(t, x_{m}, 0\right)}{c}\right) e^{j \frac{4 \pi}{\lambda}\left(\widetilde{r}\left(t, x_{m}, 0\right)-r(t, 0,0)\right)} d t \\
= & \sigma(0,0) \int_{-\frac{T}{2}}^{\frac{T}{2}} s\left(t+\frac{x_{m}}{v}-\frac{2 r\left(t+\frac{x_{m}}{v}, 0,0\right)}{c}\right) \\
& \cdot s^{*}\left(t+\frac{x_{m}}{v}-\frac{2 \widetilde{r}(t, 0,0)}{c}\right) \\
& \cdot e^{j \frac{4 \pi}{\lambda}\left(\widetilde{r}(t, 0,0)-r(t, 0,0)+r(t, 0,0)-r\left(t+\frac{x_{m}}{v}, 0,0\right)\right)} d t \\
\approx & \sigma(0,0) e^{-j \frac{2 \pi x_{m}^{2}}{\lambda R_{c}}} \int_{-\frac{T}{2}}^{\frac{T}{2}} e^{j \frac{4 \pi}{\lambda}(\widetilde{r}(t, 0,0)-r(t, 0,0))} e^{-j 2 \pi \frac{2 v x_{m}}{\lambda R_{c}} t} d t
\end{aligned}
$$

where $r\left(t+\left(x_{m} / v\right), x_{m}, 0\right)=r(t, 0,0), \widetilde{r}\left(t+\left(x_{m} / v\right), x_{m}, 0\right)=$ $\widetilde{r}(t, 0,0)$, and $s(t-((2(r(t, 0,0))) / c)) \approx s(t-$ $\left.\left(\left(2 r\left(t, x_{m}, 0\right)\right) / c\right)\right) \approx s\left(t-\left(\left(2 \widetilde{r}\left(t, x_{m}, 0\right)\right) / c\right)\right) \approx$ $s(t-((2 \widetilde{r}(t, 0,0)) / c))$.

From (17) and (18), the PCD imaging error can be derived as

$$
\begin{aligned}
& \varepsilon^{2} \\
& =\frac{\int_{-\infty}^{\infty}\left|\widetilde{I}\left(x_{m}, 0\right)-I\left(x_{m}, 0\right)\right|^{2} d x_{m}}{\int_{-\infty}^{\infty}\left|I\left(x_{m}, 0\right)\right|^{2} d x_{m}} \\
& =\frac{\int_{-\infty}^{\infty}\left|\int_{-\frac{T}{2}}^{\frac{T}{2}}\left(e^{j \frac{4 \pi}{\lambda}(\widetilde{r}(t, 0,0)-r(t, 0,0))}-1\right) e^{-j 2 \pi \frac{2 v x_{m}}{\lambda R_{c}} t} d t\right|^{2} d \frac{2 v x_{m}}{\lambda R_{c}}}{\int_{-\infty}^{\infty}\left|\int_{-\frac{T}{2}}^{\frac{T}{2}} e^{-j 2 \pi \frac{2 v x_{m}}{\lambda R_{c}} t} d t\right|^{2} d \frac{2 v x_{m}}{\lambda R_{c}}} \\
& =\frac{1}{T} \int_{-\frac{T}{2}}^{\frac{T}{2}}\left|e^{j \frac{4 \pi}{\lambda}(\widetilde{r}(t, 0,0)-r(t, 0,0))}-1\right|^{2} d t .
\end{aligned}
$$

In deriving (19), Parseval's theorem is applied, by which the power in frequency domain with respect to $\left(2 v x_{m} / \lambda R_{c}\right)$ is equal to that in time domain with respect to $t$. From (1) and (6), since $\widetilde{r}(t, 0,0)-r(t, 0,0)$ can be approximated as a function with period $T_{P}=T / P$, (19) can be further derived as

$$
\begin{aligned}
\varepsilon^{2} & =\frac{1}{T_{P}} \int_{t_{p}}^{t_{p+1}}\left|e^{j \frac{4 \pi}{\lambda}(\widetilde{r}(t, 0,0)-r(t, 0,0))}-1\right|^{2} d t \\
& =2-2 \operatorname{Re}\left\{\frac{1}{T_{P}} \int_{t_{p}}^{t_{p+1}} e^{j \frac{4 \pi}{\lambda}(\widetilde{r}(t, 0,0)-r(t, 0,0))} d t\right\} \\
& =2-2 \operatorname{Re}\left\{\frac{1}{T_{P}} \int_{t_{p}}^{t_{p+1}} e^{j \frac{2 \pi v^{2}}{\lambda R_{C}}\left(-t^{2}+\left(t_{p}+t_{p+1}\right) t-t_{p} t_{p+1}\right)} d t\right\} \\
& =2-2 \operatorname{Re}\left\{\frac{1}{T_{P}} \int_{t_{p}}^{t_{p+1}} e^{j \frac{2 \pi v^{2}}{\lambda R_{C}}\left(-\left(t-\frac{t_{p}+t_{p+1}}{2}\right)^{2}+\frac{T_{P}^{2}}{4}\right)} d t\right\} \\
& =2-2 \operatorname{Re}\left\{\frac{1}{T_{P}} e^{j \frac{\pi v^{2} T_{P}^{2}}{2 \lambda R_{C}}} \int_{-\frac{T_{P}}{2}}^{\frac{T_{P}}{2}} e^{-j \frac{2 \pi v^{2}}{\lambda R_{c}} t^{2}} d t\right\} \\
& =2-2 \operatorname{Re}\left\{\sqrt{\frac{\lambda R_{c}}{2 \pi v^{2} T_{P}^{2}}} e^{j \frac{\pi v^{2} T_{P}^{2}}{2 \lambda R_{C}}} \int_{-\frac{T_{P}}{2} v \sqrt{\frac{2 \pi}{\lambda R_{C}}}}^{\frac{T_{P}}{2} v \sqrt{\frac{2 \pi}{\lambda R_{C}}}} e^{-j t^{2}} d t\right\} .
\end{aligned}
$$

According to the Fresnel integrals, (20) can be expressed as

$$
\begin{aligned}
\varepsilon^{2}=2-2 \operatorname{Re}\{ & \sqrt{\frac{L_{a} P^{2}}{2 \pi L}} e^{j \frac{\pi L}{2 L_{a} P^{2}}}\left(C\left(\sqrt{\frac{\pi L}{2 L_{a} P^{2}}}\right)\right. \\
& -C\left(-\sqrt{\frac{\pi L}{2 L_{a} P^{2}}}\right)+j S\left(-\sqrt{\frac{\pi L}{2 L_{a} P^{2}}}\right) \\
=2-2 \operatorname{Re}\{ & \left.\left.\sqrt{\frac{2 L_{a} P^{2}}{\pi L}} e^{j \frac{\pi L}{2 L_{a} P^{2}}}\left(\sqrt{\frac{\pi L}{2 L_{a} P^{2}}}\right)\right)\right\}\left(\sqrt{\frac{\pi L}{2 L_{a} P^{2}}}\right) \\
& \left.\left.-j S\left(\sqrt{\frac{\pi L}{2 L_{a} P^{2}}}\right)\right)\right\}
\end{aligned}
$$


where $L L_{a}=\lambda R_{c}$ and the Fresnel integrals are odd functions expressed as

$$
S(x)=\int_{0}^{x} \sin \left(t^{2}\right) d t=\sum_{n=0}^{\infty}(-1)^{n} \frac{x^{4 n+3}}{(2 n+1) !(4 n+3)}
$$

and

$$
C(x)=\int_{0}^{x} \cos \left(t^{2}\right) d t=\sum_{n=0}^{\infty}(-1)^{n} \frac{x^{4 n+1}}{(2 n) !(4 n+1)} .
$$

After defining the quality factor $Q$, (21) becomes (15).

\section{REFERENCES}

[1] P. Berardino, G. Fornaro, R. Lanari, and E. Sansosti, "A new algorithm for surface deformation monitoring based on small baseline differential SAR interferograms," IEEE Trans. Geosci. Remote Sens., vol. 40, no. 11, pp. 2375-2383, Nov. 2002.

[2] A. Rosenqvist, M. Shimada, N. Ito, and M. Watanabe, "ALOS PALSAR: A pathfinder mission for global-scale monitoring of the environment," IEEE Trans. Geosci. Remote Sens., vol. 45, no. 11, pp. 3307-3316, Nov. 2007.

[3] C. Clemente and J. J. Soraghan, "Vibrating target micro-Doppler signature in bistatic SAR with a fixed receiver," IEEE Trans. Geosci. Remote Sens., vol. 50, no. 8, pp. 3219-3227, Aug. 2012.

[4] Y. Nan, X. Huang, and Y. J. Guo, "Generalized continuous wave synthetic aperture radar for high resolution and wide swath remote sensing," IEEE Trans. Geosci. Remote Sens., vol. 56, no. 12, pp. 7217-7229, Dec. 2018.

[5] Y. Nan, X. Huang, and Y. J. Guo, "A generalized continuous wave synthetic aperture radar," in Proc. IEEE 85th Veh. Technol. Conf. (VTC Spring), Jun. 2017, pp. 1-5.

[6] Y. Nan, X. Huang, and Y. J. Guo, "Passive synthetic aperture radar imaging with piecewise constant Doppler algorithm," in Proc. IEEE-APS Top. Conf. Antennas Propag. Wireless Commun. (APWC), Sep. 2017, pp. $41-44$

[7] A. T. Le, Y. Nan, L. C. Tran, X. Huang, Y. J. Guo, and Y. Vardaxoglou, "Analog least mean square loop for self-interference cancellation in generalized continuous wave SAR," in Proc. 88th IEEE Veh. Technol. Conf. (VTC Spring), Chicago, IL, USA, Aug. 2018, pp. $1-5$.

[8] I. G. Cumming and F. H. Wong, Digital Processing of Synthetic Aperture Radar Data, vol. 1, no. 2. Norwood, MA, USA: Artech House, 2005.

[9] A. Sabharwal, P. Schniter, D. Guo, D. W. Bliss, S. Rangarajan, and R. Wichman, "In-band full-duplex wireless: Challenges and opportunities," IEEE J. Sel. Areas Commun., vol. 32, no. 9, pp. 1637-1652, Sep. 2014

[10] X. Huang and Y. J. Guo, "Radio frequency self-interference cancellation with analog least mean-square loop," IEEE Trans. Microw. Theory Techn., vol. 65, no. 9, pp. 3336-3350, Sep. 2017.

[11] A. T. Le, L. C. Tran, X. Huang, Y. J. Guo, and J. Y. C. Vardaxoglou, "Frequency-domain characterization and performance bounds of ALMS loop for RF self-interference cancellation," IEEE Trans. Commun., vol. 67 , no. 1 , pp. 682-692, Jan. 2019.

[12] A. G. Stove, "Linear FMCW radar techniques," IEE Proc. F-Radar Signal Process., vol. 139, no. 5, pp. 343-350, Oct. 1992.

[13] A. Meta, P. Hoogeboom, and L. P. Ligthart, "Signal processing for FMCW SAR," IEEE Trans. Geosci. Remote Sens., vol. 45, no. 11, pp. 3519-3532, Nov. 2007.

[14] Y. L. Neo, F. H. Wong, and I. G. Cumming, "Processing of azimuth-invariant bistatic SAR data using the range Doppler algorithm," IEEE Trans. Geosci. Remote Sens., vol. 46, no. 1, pp. 14-21, Jan. 2008.

[15] W. Wang, Z. Liu, X. Song, and X. Ma, "Research of imaging method for spaceborne mosaic mode SAR," in Proc. IEEE 11th Int. Conf. Signal Process., vol. 3, Oct. 2012, pp. 1847-1851.

[16] N. Li, R. Wang, Y. Deng, T. Zhao, W. Wang, and H. Zhang, "Processing sliding mosaic mode data with modified full-aperture imaging algorithm integrating scalloping correction," IEEE J. Sel. Topics Appl. Earth Observ. Remote Sens., vol. 10, no. 5, pp. 1804-1812, May 2017.

[17] Texas Instruments. (Dec. 2018). AWR1843 Single-Chip 77- and 79-GHz FMCW Radar Sensor. [Online]. Available: http://www.ti.com/lit/ds/ swrs222/swrs222.pdf

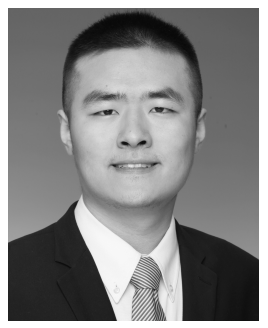

Yijiang Nan received the bachelor's degree from Tianjin University, Tianjin, China, in 2013, and the master's degree from the Beijing University of Posts and Telecommunications (BUPT), Beijing, China, in 2016. He is currently pursuing the Ph.D. degree in engineering with the Global Big Data Technologies Center, University of Technology Sydney (UTS), Sydney, NSW, Australia.

His research interests include radar signal processing, radar imaging techniques, cognitive radios, and statistical signal processing.

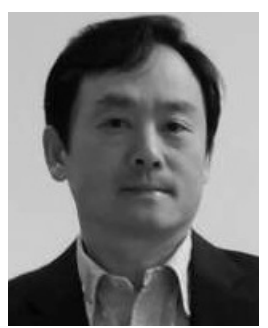

Xiaojing Huang (M'99-SM'11) received the B.Eng., M.Eng., and Ph.D. degrees in electronic engineering from Shanghai Jiao Tong University, Shanghai, China, in 1983, 1986, and 1989, respectively.

He was a Principal Research Engineer with the Motorola Australian Research Center, Botany, NSW, Australia, from 1998 to 2003, and an Associate Professor with the University of Wollongong, Wollongong, NSW, Australia, from 2004 to 2008. $\mathrm{He}$ has been a Principal Research Scientist with Commonwealth Scientific and Industrial Research Organisation (CSIRO), Sydney, NSW, Australia, and the Project Leader of the CSIRO Microwave and Millimeter-Wave Backhaul Projects, since 2009. He is currently a Professor of information and communications technology with the School of Electrical and Data Engineering and the Program Leader of mobile sensing and communications with the Global Big Data Technologies Center, University of Technology Sydney (UTS), Sydney. With over 30 years of combined industrial, academic, and scientific research experience, he has authored over 300 book chapters, refereed journal and conference articles, major commercial research reports, and filed 31 patents.

Dr. Huang was a recipient of the CSIRO Chairman's Medal and the Australian Engineering Innovation Award in 2012, for exceptional research achievements in multigigabit wireless communications.

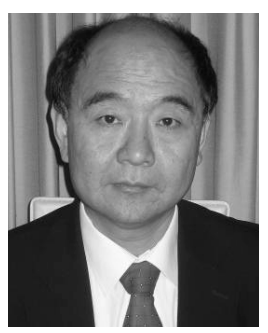

Y. Jay Guo (F'14) received the bachelor's and master's degrees from Xidian University, Xi'an, China, in 1982 and 1984, respectively, and the Ph.D. degree from Xian Jiaotong University, Xi'an, in 1987.

$\mathrm{He}$ is currently a Distinguished Professor and the Founding Director of the Global Big Data Technologies Centre (GBDTC), University of Technology Sydney (UTS), Sydney, NSW, Australia. Prior to this appointment in 2014, he served as the Director of CSIRO for over nine years. Before joining CSIRO, he held various senior technology leadership positions in Fujitsu, Siemens, and NEC, U.K. He has published over 460 research articles, including 220 journal articles, and holds 26 patents in antennas and wireless systems. His research interests include antennas, mm-wave and $\mathrm{THz}$ communications, and sensing systems as well as big data technologies.

Dr. Guo was a member of the College of Experts of Australian Research Council (ARC) from 2016 to 2018. He is a fellow of the Australian Academy of Engineering and Technology and IET. He has won a number of most prestigious Australian engineering and CSIRO awards, and was named one of the most influential engineers in Australia in 2014 and 2015, respectively. $\mathrm{He}$ has chaired numerous international conferences. He was the International Advisory Committee Chair of IEEE VTC2017, General Chair of ISAP2015, iWAT2014, and WPMC'2014, and the TPC Chair of 2010 IEEE WCNC and the 2012 and 2007 IEEE ISCIT. He is the Chair Elect of International Steering Committee and International Symposium on Antennas and Propagation (ISAP). He served as the Guest Editor for special issues on Antennas for Satellite Communications and Antennas and Propagation Aspects of 60-90 GHz Wireless Communications, in the IEEE TRANSACTIONS ON ANTENNAS AND Propagation, special Issue on Communications Challenges and Dynamics for Unmanned Autonomous Vehicles, the IEEE Journal on SELECTED AREAS IN COMMUNICATIONS (JSAC), and special Issue on $5 G$ for Mission Critical Machine Communications, the IEEE Network Magazine. 